\title{
HYDROXYAPATITE - CARBON NANOTUBE COMPOSITES FOR DRUG DELIVERY APPLICATIONS
}

\author{
Reka Barabas ${ }^{1}$, Melinda Rigo ${ }^{1}$, Melinda Sarkozi ${ }^{2}$, \\ Maria-Alexandra Hoaghia ${ }^{3}$ and Oana Cadar ${ }^{3 *}$
${ }^{1}$ Babes-Bolyai University, Faculty of Chemistry and Chemical Engineering, Cluj Napoca, Romania. ORCID: 0000-0001-6730-084X; ORCID: 0000-0003-0804-9629
${ }^{2}$ S.C. Parapharm S.R.L., Brad, Romania. ORCID: 0000-0002-9447-6821
${ }^{3}$ INCDO-INOE 2000, Research Institute for Analytical Instrumentation, Cluj-Napoca, Romania. ORCID: 0000-0001-5903-1491; E-mail: oana.cadar@icia.ro - ORCID: 0000-0002-0879-9211

(Submitted: May 4, 2018 ; Revised: October 12, 2018 ; Accepted: October 27, 2018)

\begin{abstract}
The aim of this study was the synthesis of composites containing hydroxyapatite (Hap) or silicon substituted hydroxyapatite (HapSi), carboxyl functionalized carbon nanotubes (fMWCNT) and gelatin (G) in different ratios. Ibuprofen (IBU) was chosen as a model drug for the formulation of extended-release dosage forms. The obtained composites were characterized using X-ray diffraction, laser diffraction particle size analyzer, Brunauer-Emmett-Teller surface area measurements, transmission electron microscopy and Fourier transformed infrared spectroscopy. IBU adsorption and desorption was monitored by UV-VIS spectroscopy. The obtained results revealed that composites containing three components exhibit higher adsorption efficiency (Hap-fMWCNT-20G - $82.7 \%$ and HapSi-fMWCNT-20G - 84.6\%) and extended-release of IBU, due to the chemical bonds between the carboxyl groups of IBU and the functional groups on the composite surface. The adsorption capacity of Hap composites is important for dental or orthopedic implants, the anti-inflammatory substances being adsorbed on their surface; but the adsorption capacity also enhances new bone formation (osteosynthesis) around the implants. These composites are thus attractive materials to be used in bone tissue engineering and drug delivery systems.

Keywords: Hydroxyapatite; Composite; Ibuprofen; Adsorption; Desorption.
\end{abstract}

\section{INTRODUCTION}

One of the challenges of modern medicine is the development of new materials that can mimic human tissue in order to minimize the risk of rejection by the human body [Constanda et al., 2016]. Hydroxyapatite (Hap) is a nontoxic, non-allergenic, non-mutagenic, osteoconductive, bioactive and biodegradable material with applications like coatings on different types of implant and in drug delivery systems [Cziko et al., 2016; Descamps et al., 2009; Nasiri-Tabrizi and Fahami, 2013; Tomoda et al., 2010; Wang et al., 2015]. The synthetized Hap have similar chemical composition and crystal structure to Hap in the human skeletal system. Calcium and phosphate ions, the two main constituents of hydroxyapatite, can be replaced by different types and amount of ions [Barabas et al., 2013, 2015; Cziko et al., 2016]. Various morphologies and surface properties of Hap have been studied as a drug carrier for the delivery of different pharmaceuticals [Al-Sokanee et al., 2009]. The addition of silicon (Si) into Hap enhances the speed and quality of the bone repair process. Furthermore, in vitro cell culture work showed that these SiHA thin coatings exhibited enhanced bioactivity and biofunctionality [Bang et al., 2011]. HapSi can be used as drug delivery systems and coatings for implants, while the silicon is an agent

\footnotetext{
* Corresponding author: Oana Cadar - E-mail: oana.cadar@icia.ro
} 
for potential cancer therapy [Hijon et al., 2006; Szurkowska et al., 2017].

However, recent studies have highlighted the weaknesses of Hap implants or coatings caused by its fragility, low fracture toughness, wear and corrosion resistance and negative tissue reaction. All these limitations can induce implant loosening [Constanda et al., 2016]. Therefore, in order to improve the mechanical properties and to increase the corrosion resistance of Hap or HapSi, its structure must be reinforced with various materials such as carbon nanotubes (CNTs), $\mathrm{TiO}_{2}$ or $\mathrm{ZrO}_{2}$ [Barabas et al., 2015; Park et al., 2018]. Among these reinforcements, the excellent mechanical, electrical and thermal properties make CNTs good candidates for the enhancements of Hap-based nanocomposites [Li et al., 2017]. Despite enormous potential for medical applications (cancer, sclerosis, drug delivery, etc.), their toxicity still represents a major challenge: numerous conflicting studies demonstrated both toxic and non-toxic behavior [Abrishamchian et al., 2013; Madani et al., 2013; Pistone et al., 2014; Visalli et al., 2017]. As-synthesized CNTs are agglomerated because of the strong van der Waals forces. Therefore, achieving well-dispersed suspension of CNTs in water or organic solvents, without physical or chemical modification of CNTs is challenging, but important for various applications [Abrishamchian et al., 2013; Rahman et al., 2014]. The carboxyl-functionalized CNTs have similar features and size with nanoscale collagen fibers of human bones, while the gelatin $(G)$ has the same chemical composition but requires the help of reinforcement filler [Chao et al., 2015]. Furthermore, due to their high specific surfaces and biocompatibility, the G/carboxyl-functionalized CNTs (fMWCNT) composites can be used as drug delivery systems [Yoon et al., 2014].

Recently, considerable effort has been devoted to developing novel drug release systems with improved properties (enhanced bioavailability, higher efficacy and safety, controlled and prolonged release time and predictable therapeutic effect). An efficient delivery system should be able to transport the targeting drugs to the desired cells or tissues and to release the drug in a controlled manner [Slowing et al., 2007]. Furthermore, the drug delivery carriers should be biodegradable and biocompatible, properties that depend on the drug solubility, system $\mathrm{pH}$, biological activity and the environmental chemical influence $[\mathrm{Gu}$ et al., 2014]. The time of drug release depends on both the bonds between drugs and carrier molecules, as well as the separation techniques [Xie et al., 2015]. The extended and steady drug release depends on the agents surface properties, which can be influenced by choosing the suitable preparation method [Dai et al., 2015; Tomoda et al., 2010]. In the case of IBU, good results were obtained using mesoporous materials, like Hap nanoparticles [Aghaei et al., 2014].
Ibuprofen (IBU) is a nonsteroidal anti-inflammatory drug which reduces inflammation, relieves fever and pain by blocking enzymes and protein from the human body [Bergese et al., 2015]. IBU has been frequently used as a model drug for the purpose of sustained and controlled release due to the short biological half-life $(2 \mathrm{~h})$, suitable molecule size $(\sim 0.6-1.0 \mathrm{~nm})$ and good pharmaceutical activity [Safi et al., 2018]. Its adsorption on activated carbon surface is an endothermic process, but the adsorption mechanism is not entirely elucidated [Sellaoui et al., 2015].

The combination of organic and inorganic components allows obtaining materials with good biocompatibility and osteoconductivity. The multicomponent Hap-based composites exhibit improved properties compared to their individual components [Hossan et al., 2015; Le et al., 2015; Constanda et al. 2016]. To the best of our knowledge, no studies regarding three-component Hap-based composites were reported in the literature. Therefore, the aim of this work was the synthesis and characterization of Hap/HapSi-fMWCNT-G composites by incorporation of 10 wt. $\%$ fMWCNT and different amounts of G (5, 10, 15 and $20 \mathrm{wt} \%$ ) into Hap/HapSi, in order to mimic the collagen - Hap bonds in the human skeleton. The multi-component composites of Hap/HapSi, fMWCNT and $\mathrm{G}$ are expected to have higher potential for different applications due to the excellent combination of properties (e.g., enhanced biocompatibility provided by Hap, extended drug release due to Si, reinforcing effect provided by fMWCNT, plasticity and adhesiveness due to $\mathrm{G}$, etc.). The structural characteristics of Hap-fMWCNT-G composites were investigated by X-ray diffraction (XRD), Fourier transform infrared spectroscopy (FT-IR), transmission electron microscopy (TEM) and surface area analysis (BET), whereas the adsorption properties of these composites were investigated using IBU as a model drug. The obtained composites have tremendous potential for use in bone regenerative applications.

\section{MATERIALS AND METHODS}

\section{Materials}

All reagents were of analytical grade (Merck, Germany) and used as received without further purification, except gelatin (type $\mathrm{B}, \mathrm{Mw}=25,000$ ) that was purchased from Sigma-Aldrich, Germany. fMWCNTs with specific surface of $30 \mathrm{~m}^{2} / \mathrm{g}$ and pore volume of $0.1 \mathrm{~cm}^{3} / \mathrm{g}$ were synthetized by chemical vapor deposition technique according to Barabas et al. (2015).

\section{Preparation of Hap and HapSi composites}

The composites were prepared by a precipitation method in a similar way as the synthesis of pure Hap 
using $0.5 \mathrm{~mol} / \mathrm{L} \mathrm{Ca}\left(\mathrm{NO}_{3}\right)_{2} \cdot 4 \mathrm{H}_{2} \mathrm{O}$ as source of $\mathrm{Ca}^{2+}$ and $0.3 \mathrm{~mol} / \mathrm{L}\left(\mathrm{NH}_{4}\right)_{2} \mathrm{HPO}_{4}$ as source of $\mathrm{PO}_{4}^{3-}$ (Table 1, Fig. 1) [Barabas et al., 2016]. In the case of composites containing fMWCNT and/or G, the additives were added to the aqueous solution of $\mathrm{Ca}\left(\mathrm{NO}_{3}\right)_{2} \cdot 4 \mathrm{H}_{2} \mathrm{O}$ (fMWCNT - ultrasonic treatment and intensive stirring for $10 \mathrm{~min}$ and $\mathrm{G}-\mathrm{G}$ dissolved in hot distilled water). The $\mathrm{pH}$ was adjusted to 11 by adding a $25 \%$ ammonia solution under constant stirring at room temperature. The composites containing HapSi (10 wt. \% Si) were prepared in a similar way, by adding an aqueous of $\mathrm{Na}_{2} \mathrm{SiO}_{3}$ (Lach-Ner, Czech Republic) together with $\left(\mathrm{NH}_{4}\right)_{2} \mathrm{HPO}_{4}$. The mixture was mixed for $22 \mathrm{~h}$, the precipitate was filtered, washed with distilled water and dried for 24 hours, at $105^{\circ} \mathrm{C}$, in air.

Table 1. Synthesis route of Hap and HapSi composites.

\begin{tabular}{ccccc}
\hline No & Hap/HapSi & $\begin{array}{c}\mathbf{f M W C N T} \\
(\mathbf{w t} \%)\end{array}$ & $\begin{array}{c}\mathbf{G} \\
(\mathbf{w t} \%)\end{array}$ & Composite \\
\hline 1 & Hap & 10 & 0 & Hap-fMWCNT \\
2 & Hap & 10 & 5 & Hap-fMWCNT-5G \\
3 & Hap & 10 & 10 & Hap-fMWCNT-10G \\
4 & Hap & 10 & 15 & Hap-fMWCNT-15G \\
5 & Hap & 10 & 20 & Hap-fMWCNT-20G \\
6 & Hap & 0 & 5 & Hap-5G \\
7 & HapSi & 10 & 0 & HapSi-fMW-CNT \\
8 & HapSi & 10 & 5 & HapSi-fMWCNT-5G \\
9 & HapSi & 10 & 10 & HapSi-fMWCNT-10G \\
10 & HapSi & 10 & 15 & HapSi-fMWCNT-15G \\
11 & HapSi & 10 & 20 & HapSi-fMWCNT-20G \\
12 & HapSi & 0 & 5 & HapSi-5G \\
\hline
\end{tabular}

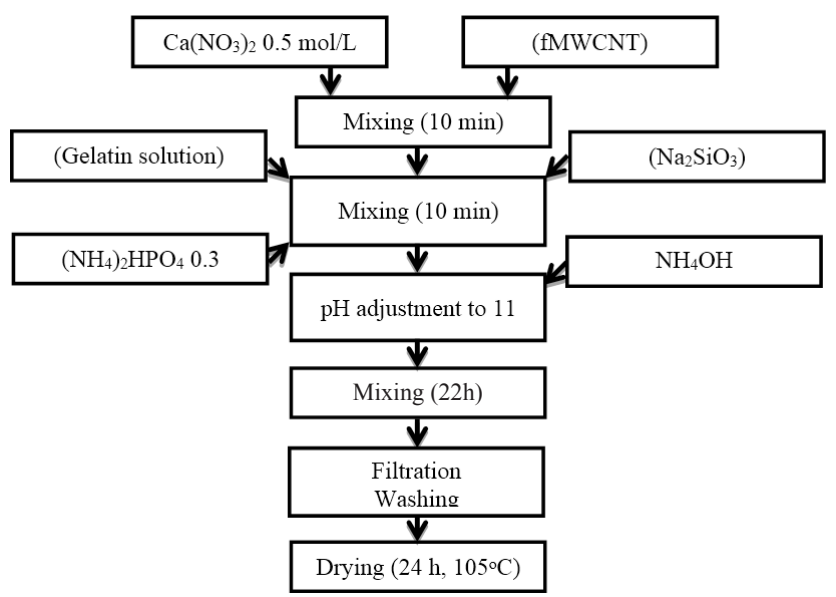

Figure 1. Schematic procedure of Hap and HapSi composites synthesis.

\section{Adsorption of IBU}

The composites were ground using a mortar and pestle, followed by the preparation of ibuprofen solution $(17 \mathrm{~g} / \mathrm{L}$ IBU is the concentration that grants a maximum adsorption) [Chavalier et al., 2010]. A mixture of $0.1 \mathrm{~g}$ composite and $0.5 \mathrm{ml}$ ethanolic IBU solution $(17 \mathrm{~g} / \mathrm{L})$ was stirred for $22 \mathrm{~h}$. The solid phase was separated by centrifugation and dried at $40-50{ }^{\circ} \mathrm{C}$ for $24 \mathrm{~h}$. The concentration of IBU was measured in both liquid and solid phase by UV-VIS spectrophotometry, at $272 \mathrm{~nm}$. The pellets with a diameter of $130 \mathrm{~mm}$ were prepared using a Specac Atlas 15T manual hydraulic press.

\section{IBU desorption}

The release behavior of the IBU was investigated by soaking each tablet in $50 \mathrm{~mL}$ of simulated body fluid (SBF, $\mathrm{pH}=7.4$ ) at $37^{\circ} \mathrm{C}$, for $24 \mathrm{~h}$ [Kokubo et al., 1990; Moisa et al., 2018]. $0.45 \mu \mathrm{m}$ membrane filters were used to avoid interference from suspended particles in the UV-VIS analysis. A $10 \mathrm{~mL}$ sample was removed at given intervals and analyzed by using a UV-VIS spectrophotometer at $272 \mathrm{~nm}$. The volume removed was replaced with the same amount of preheated SBF. The experiments were carried-out in triplicate.

\section{Characterization}

- XRD analysis was performed at room temperature, using a D8 Advance diffractometer (Bruker, Germany), operating at $40 \mathrm{kV}, 40 \mathrm{~mA}$ with $\mathrm{CuK}_{\alpha}$ radiation $(\lambda=1.54060 \AA)$.

- $\quad$ The FT-IR spectra were recorded using a FT/ IR-615 spectrophotometer (Jasco, Japan).

- The specific surface area of samples was determined using Asap 2000 equipment (Micromeritics Instruments Inc., USA), from data on nitrogen adsorption.

- The particle size of composites in suspension was determined using a particle size analyzer SALD7101 (Shimadzu, Japan).

- The morphology of composites was observed by a H7650 transmission electron microscope (TEM) (Hitachi, Japan).

- The adsorption-desorption IBU studies were performed using a V-560 UV-VIS spectrophotometer (Jasco, Japan).

\section{RESULTS AND DISCUSSION}

\section{X-ray diffraction (XRD)}

The XRD patterns of Hap and HapSi composites (Fig. 2) show the characteristic diffraction lines for pure Hap (JCPDS file No. 09-0432). The peak observed at $25.8^{\circ}$ attributed to CNTs overlaps with the peak of pure Hap [Zhao et al., 2010]. The degree of crystallinity of composites decreased slightly with the increase of $G$ content, supporting the presence of interactions between Hap and G. Furthermore, in general, the degree of crystallinity depends also on thermal treatment conditions [Bang et al., 2011]. In our experiments, all the thermal conditions were the same; therefore, it can be concluded that the increase of degree of crystallinity of composites can be attributed to the presence of G. Poorly crystalline Hap and HapSi phases were formed in the case of two-component composites (Hap/HapSi, fMWCNT or G). 


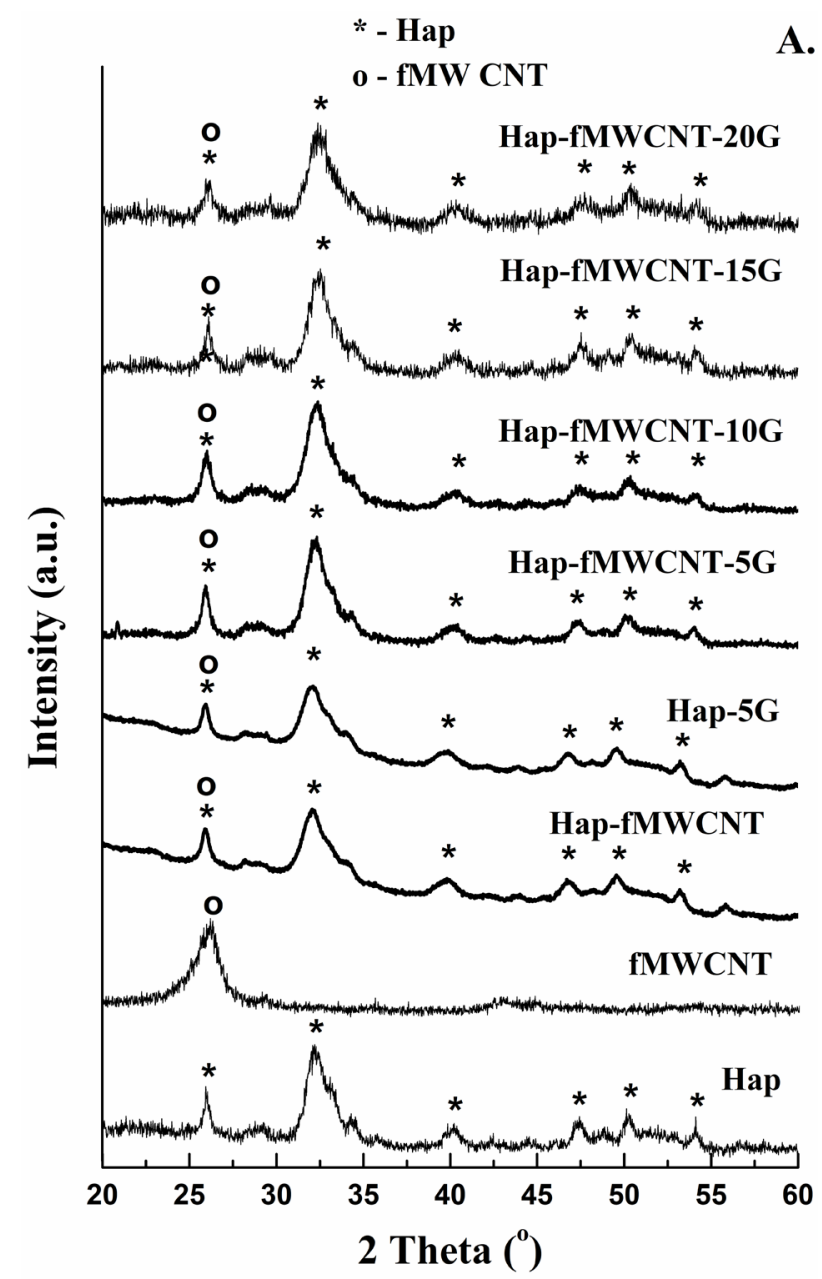

A.

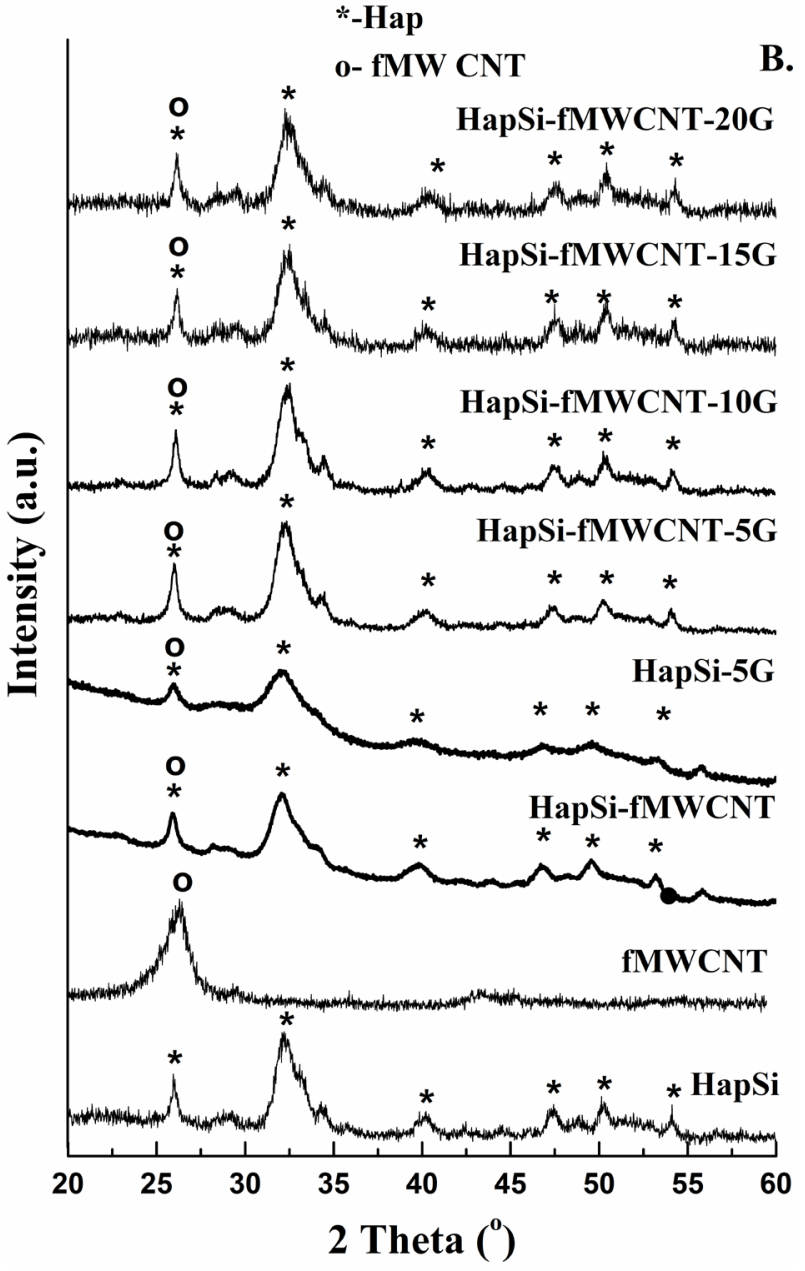

Figure 2. XRD patterns of Hap (A) - and HapSi (B) - composites.

\section{Laser diffraction particle size analyzer}

Fig. 3 shows the average particle size of Hap (a) and HapSi (b) composites. The proteins play a significant role during the initial nucleation of biomineral crystals and their subsequent growth, the proteins acting to inhibit or stimulate nucleation and growth [Tarasevich et al., 2007]. $G$ is a crystallization inhibitor, which reduces the growth rate even at low $\mathrm{G}$ concentration [Sun et al., 2007]. The average particle size decreases

A.

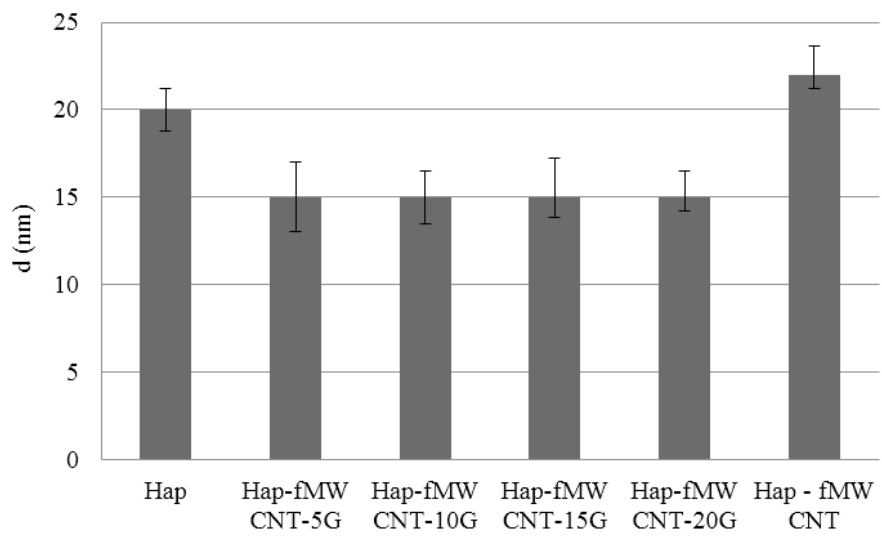

in Hap composites containing G (until $15 \mathrm{~nm}$ ). For HapSi, the G effect appears only above $10 \%$ content $(24 \mathrm{~nm}-5 \%, 15 \mathrm{~nm}-10 \%, 15 \mathrm{~nm}-15 \%$ and $17 \mathrm{~nm}-$ $20 \%$, respectively).

\section{Specific surface area determination (BET)}

The specific surface area is an important factor for the evaluation of the chemical activity and adsorption capacity of materials [Tantra, 2016]. The specific surface

B.

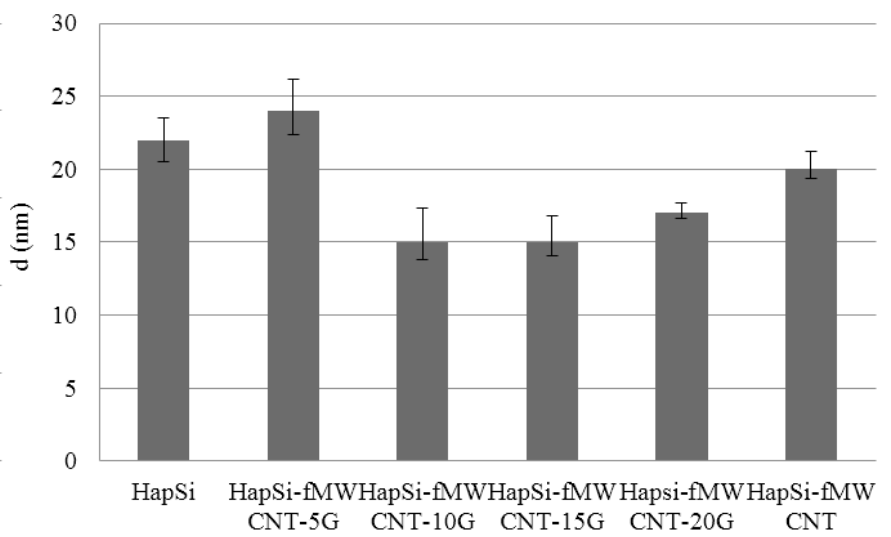

Figure 3. Average particle size of Hap (A) - and HapSi (B) composites. 
area decreased upon adding $\mathrm{SiO}_{2}$ to hydroxyapatite (Fig. 4). $\mathrm{SiO}_{2}$ is acting more like a binder, due to both the polymerization of excess $\mathrm{SiO}_{2}$ and the interactions with functional groups of Hap, G and fMWCNT from the system [Putlayev et al., 2006]. The highest specific surface area was determined for Hap-fMWCNT-10G $\left(150 \mathrm{~m}^{2} / \mathrm{g}\right)$ and Hap-fMWCNT-15G $\left(140 \mathrm{~m}^{2} / \mathrm{g}\right)$.

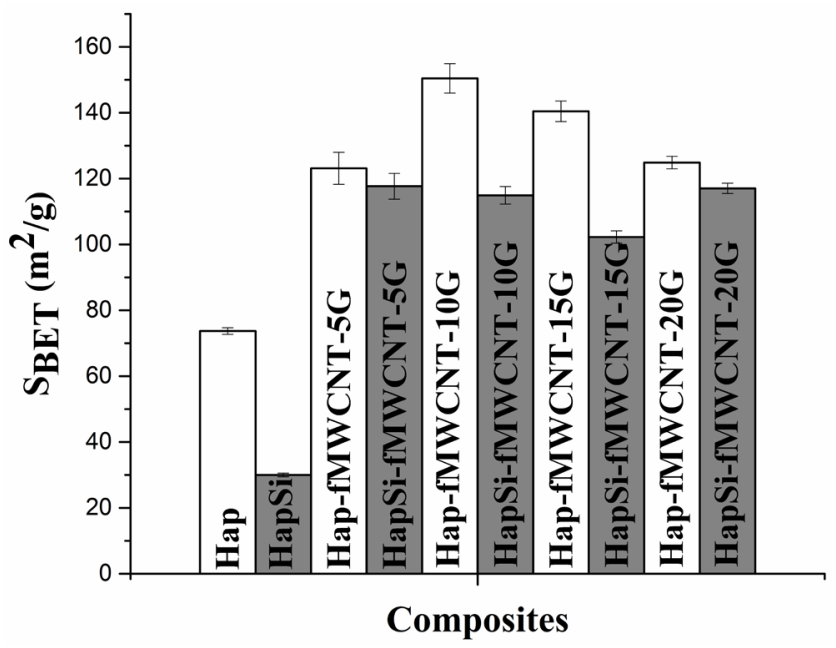

Figure 4. Specific surface area of Hap (white) and HapSi (grey) composites.

\section{Transmission electron microscopy (TEM)}

TEM images of Hap and HapSi composites are presented in Fig. 5 (a-1). TEM images of composites containing $\mathrm{G}(\mathrm{a}-\mathrm{h})$ revealed no fMWCNT, resulting in a high homogenization degree. Only for composites containing $20 \% \mathrm{G}$ ( $\mathrm{g}$ and $\mathrm{h}$ ), the presence of $\mathrm{G}$ was evidenced. As observed, the $G$ has a stick effect on the particles (Fig. 5d, e and g), while in the Hap-G composites, $G$ has a strong influence on particle morphology (spherical particles) (Fig. 5k). The addition of Si to Hap composites results in a higher degree of agglomeration, Si acting as a binder material (Fig. $5 \mathrm{i}$ and j) [Cziko et al., 2016].

\section{FT-IR analysis}

Fig. 6 presents the FT-IR spectra of Hap-fMWCNT$20 \mathrm{G}$ and HapSi-fMWCNT-20G and IBU absorbed composites. The spectra are similar due to the low concentration of IBU. The characteristic bands of Hap (P-O), G (C-C, amino and hydroxyl group), fMWCNT (carboxyl groups) and IBU (carboxyl groups) were observed. The specific band of aliphatic amine bonds at $1020 \mathrm{~cm}^{-1}$ confirms the presence of $\mathrm{G}$, while the specific band of C-C cyclic bonds at 1417-1442 $\mathrm{cm}^{-1}$ confirms the presence of fMWCNT in the composites (Table 1) (Stuart, 2004).

\section{Ultraviolet-visible spectroscopy (UV-VIS)}

The adsorption and desorption efficiency of Hap and HapSi composites was investigated by UV-VIS (a) Hap-fMWCNT-5G

(b) HapSi-fMWCNT-5G

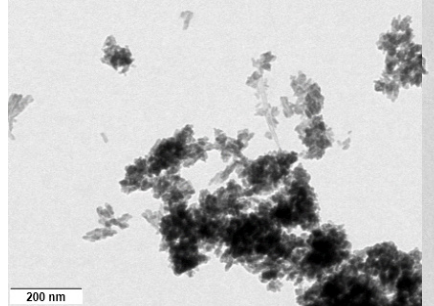

(c) Hap-fMWCNT-10G

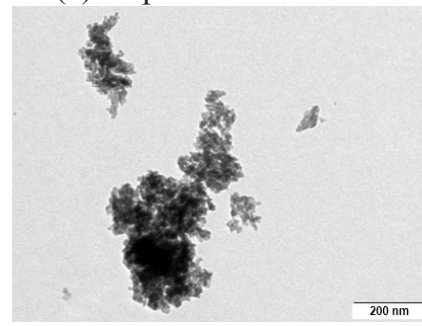

(e) Hap-fMWCNT-15G

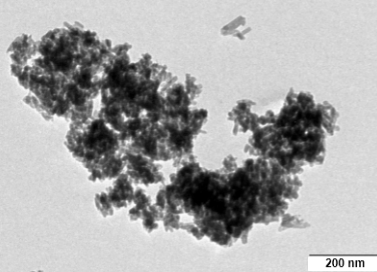

(d) HapSi-fMWCNT-10G

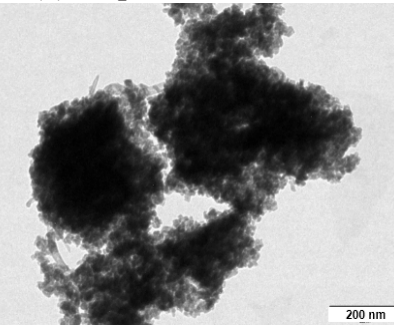

(g) Hap-fMWCNT-20G

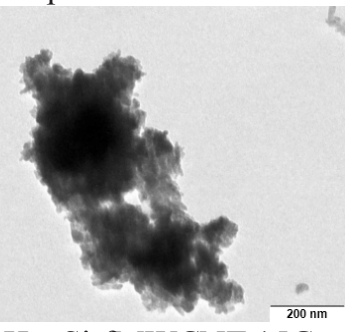

(f) HapSi-fMWCNT-15G

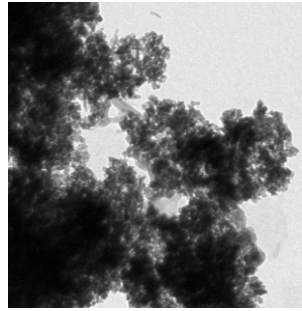

(h) HapSi-fMWCNT-20G

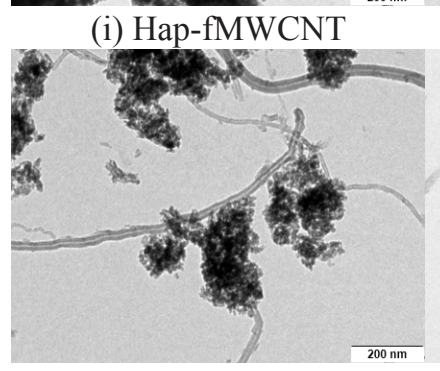

(k) Hap-5G
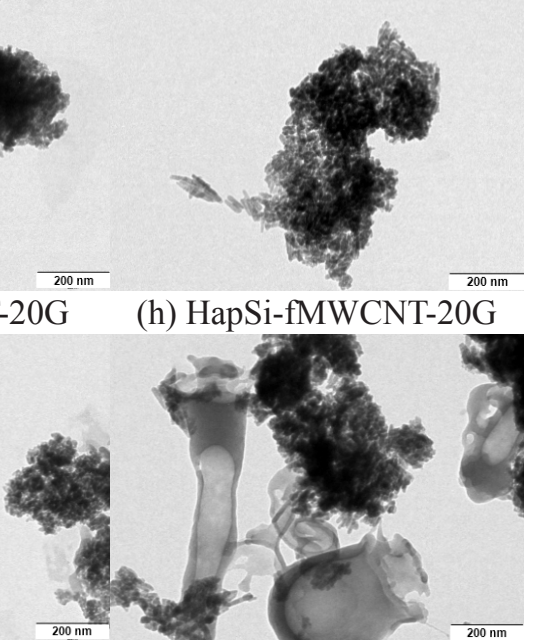

(j) HapSi-fMWCNT
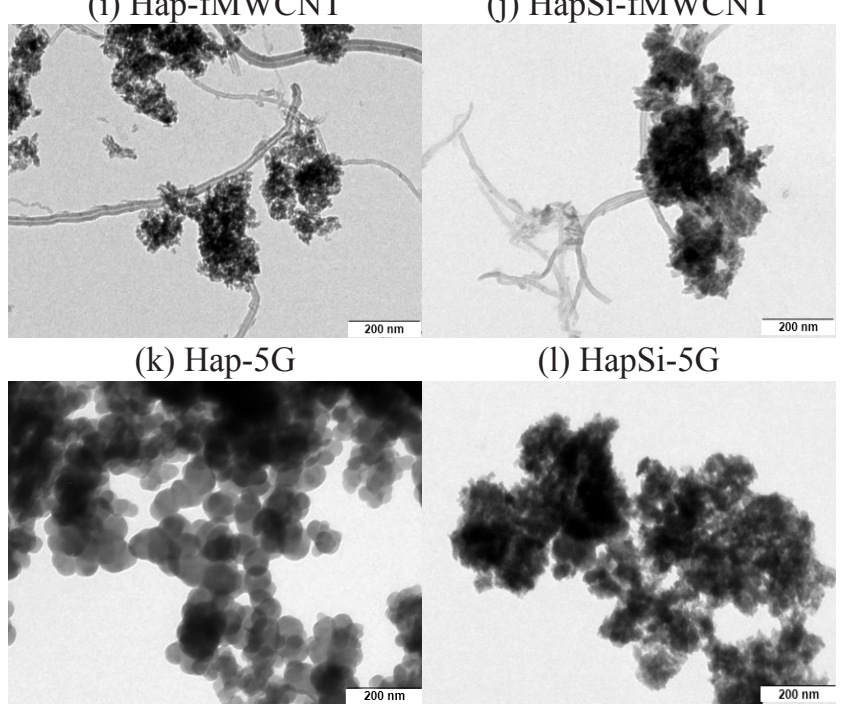

(1) HapSi-5G

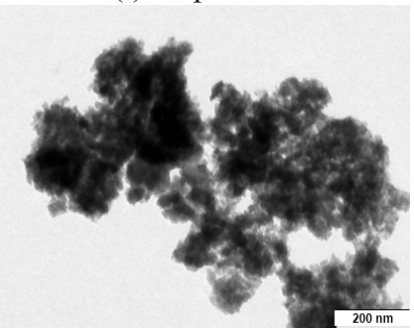

Figure 5. TEM images of Hap and HapSi composites.

spectroscopy; the adsorption experiments were carried out on both liquid and solid (powder) phases. For all the measurements, the adsorption capacity (Q) and 

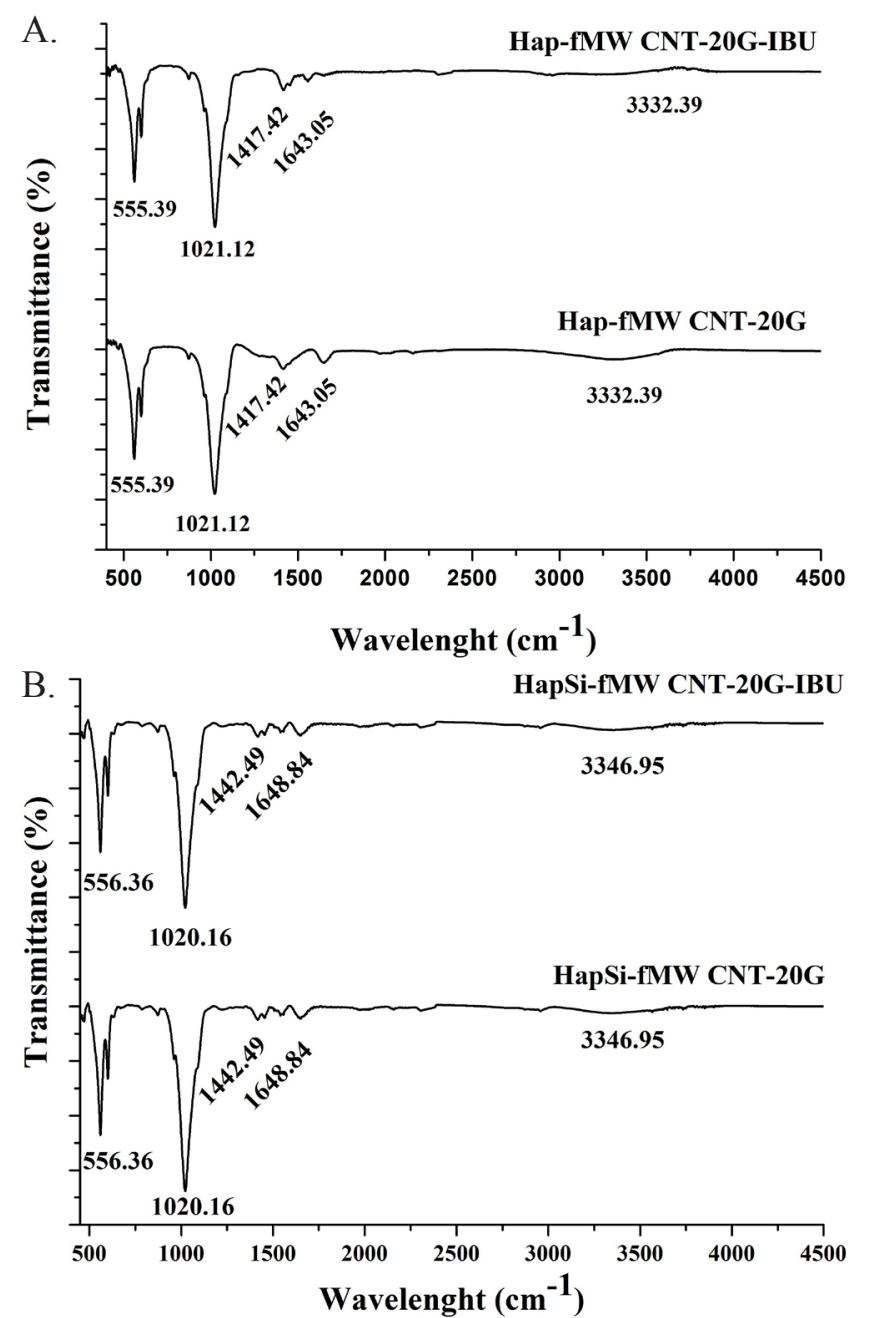

Figure 6. FT-IR spectra of Hap-fMWCNT-20G and Hap-fMWCNT-20G-IBU (A) and HapSi-fMWCNT20G and HapSi-fMWCNT-20G-IBU (B) composites.

the efficiency $(\eta)$ were calculated with the following equations:

$\mathrm{Q}=\frac{\mathrm{m}_{\mathrm{ads}}}{\mathrm{m}}(\mathrm{g} / \mathrm{g})$

$\eta=\frac{c_{o}-c_{i}}{c_{o}} \times 100(\%)$

where: $\mathrm{m}_{\text {ads }}$ - adsorbed quantity of IBU (mg), $\mathrm{m}$ quantity of composite $(\mathrm{g}), \mathrm{c}_{\mathrm{o}}$ - initial IBU concentration (initial IBU concentration, mol/L) and $\mathrm{c}_{i}-$ IBU concentration at specific time, $\mathrm{mol} / \mathrm{L}$ ).

\section{Liquid adsorption}

Fig. 7 shows the adsorption efficiency of Hap and HapSi composites. These measurements were performed in liquid phase. Above $15 \%$ G content, a sharp increase in the adsorption capacity is observed. The highest adsorption capacity was obtained for the composites containing 20\% G (Hap-fMWCNT$20 \mathrm{G}-82.7 \%$ and HapSi-fMWCNT-20G - 84.6\%). During the IBU adsorption process, several competing processes such as physical adsorption (physisorption) and chemical adsorption (chemisorption) can occur. Therefore, the adsorption depends not only on the specific surface, but also on the bonds between the functional groups of IBU and the functional groups on the composite surface: for example, the hydrogen bonds between the carboxyl group of IBU and the hydroxyl groups of G [Bach et al., 2013]. There is no obvious interdependence between the amount of $\mathrm{G}$ and the adsorption efficiency. The high adsorption efficiency of $\mathrm{Hap}(\mathrm{Si})$-fMWCNT-20G composites indicates chemisorption as the dominant process in different competitive processes and the interaction between the functional groups of G and IBU.

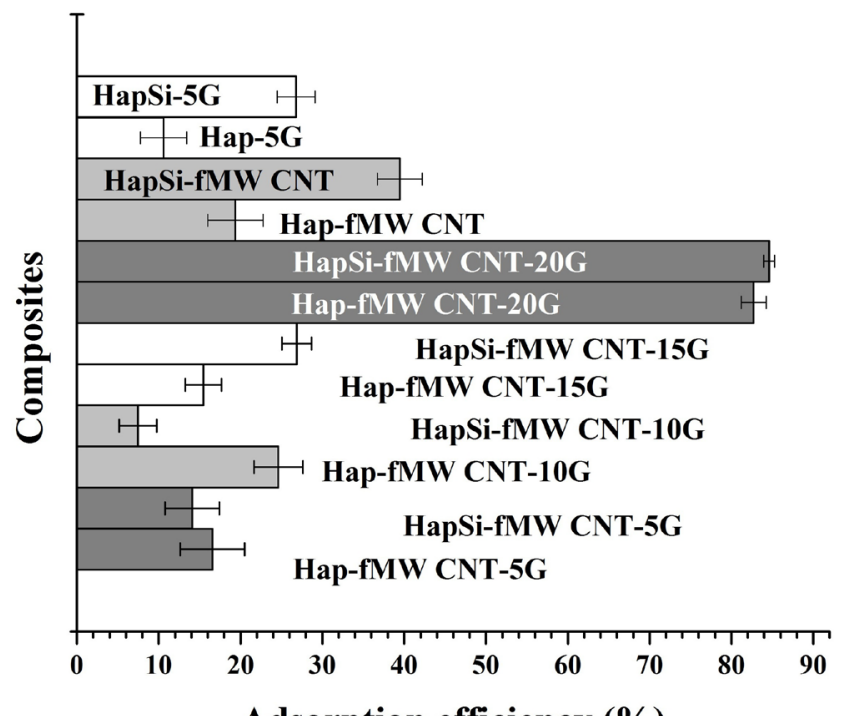

Adsorption efficiency (\%)

Figure 7. Adsorption efficiency of Hap and HapSi composites (liquid).

Table 2. FT-IR spectral assignments of Hap and HapSi composites.

\begin{tabular}{cccccc}
\hline \multirow{2}{*}{ No. } & \multirow{2}{*}{ Spectral assigments } & \multicolumn{4}{c}{ Wavenumber $\left.\mathbf{c m}^{-1}\right)$} \\
\cline { 2 - 6 } & Hap & G & fMWCNT & IBU \\
\hline 1 & P-O deformation vibration & 556 & - & - & - \\
2 & Aliphatic amine bonds & - & 1020 & - & - \\
3 & C-C cyclic bonds & - & $1417-1442$ & - & - \\
4 & Alkene, double bonds & - & 1643 & - & - \\
5 & Amino and hydroxyl groups & $3400-3500$ & $3000-3500$ & - & - \\
6 & Carboxyl groups & - & - & 3300 & 3300 \\
\hline
\end{tabular}




\section{Powder adsorption}

Fig. 8 shows the IBU absorbance at $272 \mathrm{~nm}$ of Hap and HapSi composites. The Hap and HapSi composites containing $20 \% \mathrm{G}$ and $\mathrm{MMWCNT}$ exhibit the highest adsorption efficiency (the highest absorbance value).

The obtained results of both solid and liquid phases gave good correlations. The composites containing all three components and high $\mathrm{G}$ content $(20 \%)$ exhibited the highest adsorption efficiency [Bach et al., 2013].

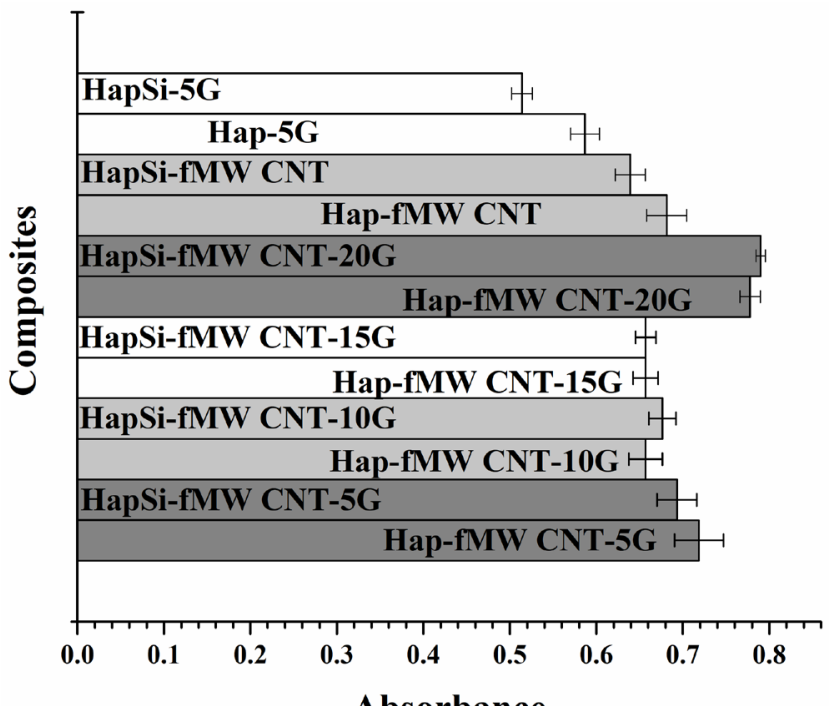

Figure 8. Adsorption capacity of Hap and HapSi composites (solid).

\section{Desorption}

In order to reveal the IBU retardation effect, its desorption from Hap and HapSi composites was performed. The IBU release from Hap and HapSi composites after soaking in SBF is shown in Fig 9. In the figure, the lines are provided for convenience. IBU release was lowest for composites containing higher G content (20\%), while the IBU absorption was the highest. Comparing the two composites, the one containing silica had a longer IBU dissolution time. A possible explanation is the polymerization process of silica in basic medium and the interaction of -OH silica bonds from silanol groups with IBU [Aghaei et al., 2014; Chevalier et al., 2010].

IBU desorption from Hap and HapSi composites is shown in Table 3. For the composites containing two components, IBU desorption is very fast (3-5 h), while for the composites with three components (Hap/HapSi, CNT and G), IBU desorption is retarded (11-24 h). For Hap/HapSi-fMWCNT-20G, the IBU desorption was not accomplished even after $24 \mathrm{~h}$ (Hap-fMWCNT20G - 35.9\% IBU and HapSi-fMWCNT-20G - 16.9 $\%$ IBU), suggesting that, for long treatments, these materials could probably be more effective as drug carriers for implant applications.

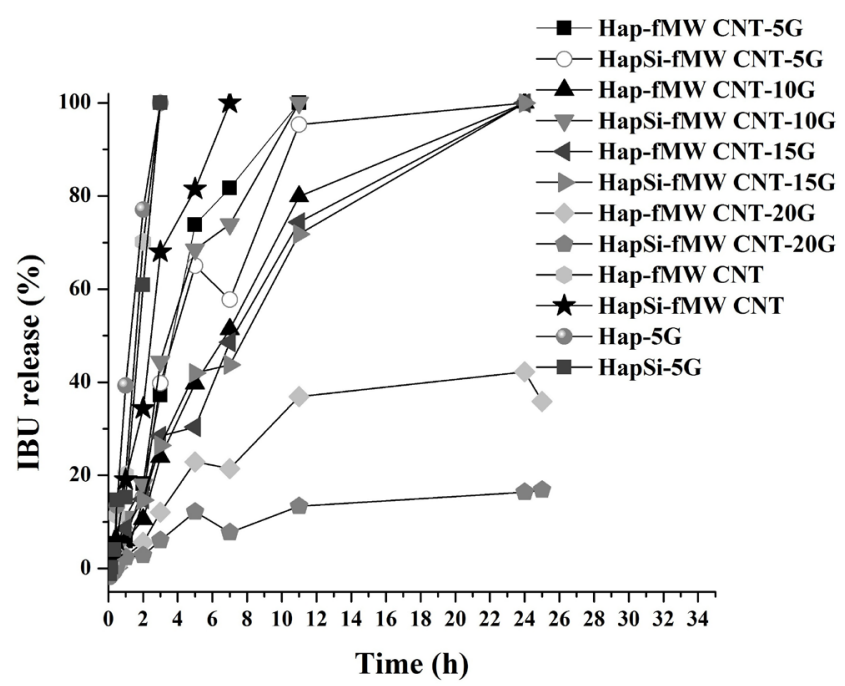

Figure 9. IBU release of Hap and HapSi composites after soaking in $\mathrm{SBF}(0-25 \mathrm{~h})$ at $37^{\circ} \mathrm{C}$.

Table 3. IBU desorption time from Hap and HapSi composites.

\begin{tabular}{cccc}
\hline Composite & $\begin{array}{c}\text { Des. } \\
\text { time (h) }\end{array}$ & Composite & $\begin{array}{c}\text { Des. } \\
\text { time (h) }\end{array}$ \\
\hline Hap-5G & $2-3$ & HapSi-5G & $2-3$ \\
Hap-fMW CNT & $2-3$ & HapSi-fMW CNT & $5-7$ \\
Hap-fMW CNT-5G & $7-11$ & HapSi-fMW CNT-5G & $11-24$ \\
Hap-fMW CNT-10G & $11-24$ & HapSi-fMW CNT-10G & $7-11$ \\
Hap-fMW CNT-15G & $11-24$ & HapSi-fMW CNT-15G & $11-24$ \\
Hap-fMW CNT-20G & - & HapSi-fMW CNT-20G & - \\
\hline
\end{tabular}

\section{CONCLUSIONS}

Composites with different Hap(Si):fMWCNT:G ratios were obtained by a precipitation method. The highest surface area was obtained for the composites with 10 and $15 \mathrm{wt} . \% \mathrm{G}$, in the absence of silica. The FT-IR spectra confirm the formation of composites. The composites containing three components (Hap/HapSi, fMWCNT and 20 wt.\% G) exhibit higher adsorption efficiency and extendedrelease of IBU, due to the chemical bonds between the carboxyl groups of IBU and the functional groups on the composite surface. The obtained composites containing IBU can be used as coatings for orthopedic implants, with tunable drug releasing properties, for after surgery inflammation. Adding G in appropriate proportion to composites containing Hap and CNT increased the adsorption efficiency, while the presence of $\mathrm{Si}$ in composites improved the extended drug release.

\section{ACKNOWLEDGEMENT}

This work was supported in part by Sectorial Operational Program "Increase of Economic Competitiveness" Priority Axis II, Project Number 1887, INOVA-OPTIMA, code SMIS-CSNR 49164. 


\section{REFERENCES}

Abrishamchian, A., Hooshmand, T., Mohammadim, M., Najafi, F. Preparation and Characterization of Multi-Walled Carbon Nanotube/Hydroxyapatite Nanocomposite Film Dip Coated on Ti-6Al-4V By Sol-Gel Method for Biomedical Applications: An in Vitro Study, Materials Science and Engineering C: Materials for Biological Applications, 33, 2002-2010 (2013). https://doi.org/10.1016/j. msec.2013.01.014

Aghaei, H., Nourbaksh, A.A., Karbasi, S., JavadKalbasi, R., Rafienia, M., Nourbakhsh, N., Bonakdar, S., Mackenzie, K.J.D. Investigation on Bioactivity and Cytotoxicity of Mesoporous Nano-Composite MCM-48/Hydroxyapatite for Ibuprofen Drug Delivery, Ceramics International, 40, 7355-7362 (2014). https://doi.org/10.1016/j. ceramint.2013.12.079

Al-Sokanee, Z.N., Toabi, A.A.H., Al-Assadi. M.J., Alassadi, E.A.S. The Drug Release Study of Ceftriaxone From Porous Hydroxyapatite Scaffolds, An Official Journal of the American Association of Pharmaceutical Scientists, 10, 772779 (2009). https://doi.org/10.1208/s12249-0099265-7

Bach, L.G., Islam, M.R., Lim, K.T. Expanding Hyperbranched Polyglycerols on Hydroxyapatite Nanocrystals via Ring-Opening Multibranching Polymerization for Controlled Drug Delivery System, Materials Letters, 93, 64-67 (2013). https:// doi.org/10.1016/j.matlet.2012.11.051

Bang, L.T., Ishikawa, K., Othman R. Effect of Silicon and Heat-Treatment Temperature on the Morphology and Mechanical Properties Of Silicon-Substituted Hydroxyapatite, Ceramics International, 37, 3637-3642 (2011). https://doi. org/10.1016/j.ceramint.2011.06.023

Barabas, R., Cziko, M., Dekany, I., Bizo, L., Bogya, E.S. Comparative Study of Particle Size Analysis of Hydroxyapatite-Based Nanomaterials, Chemical Papers, 67, 1414-1423 (2013). https://doi. org/10.2478/s11696-013-0409-6

Barabas, R., Katona, G., Bogya, E.S., Diudea, M.V., Szentes, A., Zsirka, B., Kovacs, J., Kekedy-Nagy, L., Cziko, M. Preparation and characterization of Carboxyl Functionalized Multiwall Carbon Nanotubes-Hydroxyapatite Composites, Ceramics International, 41, 12717-12727 (2015). https://doi. org/10.1016/j.ceramint.2015.06.104

Bergese, S.D., Candiotti, K., Ayad, S.S., Soghomonyan, S., Gan, T.J. The Shortened Infusion Time of Intravenous Ibuprofen Part 1: A Multicenter, OpenLabel, Surveillance Trial to Evaluate Safety and Efficacy, Clinical Therapeutics, 37, 360-367 (2015). https://doi.org/10.1016/j.clinthera.2014.12.004
Chao, S.C., Wang, M.J., Pai, N.S., Yen, S.K. Preparation and Characterization of GelatinHydroxyapatite Composite Microspheres for Hard Tissue Repair, Materials Science and Engineering: C, 15, 113-122 (2015). https://doi.org/10.1016/j. msec.2015.07.047

Chevalier, E., Viana, M., Cazalbou, S., Makein, L., Dubois, J., Chulia, D. Ibuprofen-Loaded Calcium Phosphate Granules: Combination of Innovative Characterization Methods to Relate Mechanical Strength to Drug Location, Acta Biomaterialia, 6, 266-274 (2010). https://doi.org/10.1016/j.actbio.2009.07.040

Constanda, S., Stan, M.S., Ciobanu, C.S., MotelicaHeino, M., Guegan, R., Lafdi, K., Dinischiotu, A., Predoi, D. Carbon Nanotubes-Hydroxyapatite Nanocomposites for an Improved Osteoblast Cell Response, Journal of Nanomaterials, 2016, 1-10 (2016). https://doi.org/10.1155/2016/3941501

Cziko, M., Bogya, E.S., Paisz, C., Katona, G., Konya, Z., Kukovecz, A., Barabas, R. Albumin Adsorption Study onto Hydroxyapatite-Multiwall Carbon Nanotube Based Composites, Materials Chemistry and Physics, 180, 314-325 (2016). https://doi. org/10.1016/j.matchemphys.2016.06.012

Dai, C.F., Li, S.P., Li, X.D. Synthesis of Nanostructured Methotrexate/Hydroxyapatite: Morphology Control, Growth Mechanism, and Bioassay Explore, Colloids and Surfaces B: Biointerfaces, 136, 262-271 (2015). https://doi.org/10.1016/j. colsurfb.2015.09.015

Descamps, M., Hornez, J.C., Leriche, A. Manufacture of Hydroxyapatite Beads for Medical Applications, Journal of the European Ceramic Society, 29, 369-375 (2009). https://doi.org/10.1016/j. jeurceramsoc.2008.06.008

Gu, L., Xiaomei, H., Zhenyu, W., Mesoporous Hydroxyapatite: Preparation, Drug Adsorption, and Release Properties, Materials Chemistry and Physics, 148, 153-158 (2014). https://doi. org/10.1016/j.matchemphys.2014.07.024

Hijon, N., Cabanas, M.V., Pena, J., Vallet-Regi, M. Dip Coated Silicon-Substituted Hydroxyapatite Films, Acta Biomaterialia, 2, 567-574 (2006). https://doi. org/10.1016/j.actbio.2006.05.004

Hossan, M.J., Gafur, M.A., Karim, M.M., Rana, A.A. Mechanical Properties of Gelatin Hydroxyapatite Composite for Bone Tissue Engineering, Bangladesh Journal of Scientific and Industrial Research, 50, 15-20, (2015). https://doi. org/10.3329/bjsir.v50i1.23805

Kokubo, T., Kushitani, H., Sakka, S., Kitsugi, T., Yamamuro, T. Solutions Able to Reproduce in Vivo Surface-Structure Changes in Bioactive Glass-Ceramic A-W3, Journal of Biomedical Materials Research, 24, 721-734 (1990). https:// doi.org/10.1002/jbm.820240607 
Le, H., Natesan, K., Pranti-Haran, S. Mechanical Property and Biocompatibility of Co-Precipitated Nano Hydroxyapatite-Gelatine Composites, Journal of Advanced Ceramics, 4, 237-243 (2015). https://doi.org/10.1007/s40145-015-0155-Z

Li, H., Song, X., Li, B., Kang, J., Liang, C., Wang, H., Yu, Z., Qiao, Z. Carbon Nanotube-Reinforced Mesoporous Hydroxyapatite Composites with Excellent Mechanical and Biological Properties for Bone Replacement Material Application, Materials Science and Engineering C, 77, 1078-1087 (2017). https://doi.org/10.1016/j.msec.2017.04.048

Madani, S.Y., Mandel, A., Seifalian, A.M. A Concise Review of Carbon Nanotube's Toxicology, Nano Review, 4, 1-14 (2013). https://doi.org/10.3402/ nano.v4i0.21521

Moisa, C., Vicas, L.G., Ganea, M., Levei, E.A., Cadar, O., Berce, C. Murine Studies Regarding the Tissue Intake of Different Magnesium Compounds, Farmacia, 66, 176-180 (2018).

Nasiri-Tabrizi, B., Fahami, A. Synthesis and Characterization of Fluorapatite-Zirconia Composite Nanopowders, Ceramics International, 39, 4329-4337 (2013). https://doi.org/10.1016/j. ceramint.2012.11.016

Park, J.E., Jang, Y.S., Jeon, J.G., Bae, T.S., Lee, M.H. The Effect of Multi-Walled Carbon Nanotubes/Hydroxyapatite Nanocomposites on Biocompatibility, Advanced Composite Materials, 27, 53-65 (2018). https://doi.org/10.1080/0924304 6.2017 .1374705

Pistone, A., Iannazzo, D., Panseri, S., Montesi, M., Tampieri, A., Galvagno, S. Hydroxyapatitemagnetite-MWCNT nanocomposite as a biocompatible multifunctional drug delivery system for bone tissue engineering, Nanotechnology, 25, 1-9 (2014). https://doi.org/10.1088/0957$4484 / 25 / 42 / 425701$

Putlayev, V., Veresov, A., Pulkin, M., Soin, A., Kuznetsov, V. Silicon-substituted hydroxyapatite ceramics (Si-HAp): densification and grain growth through the prism of sintering theories, Materialwissenschaft und Werkstofftechnik, 37, 416-421 (2006). https://doi.org/10.1002/ mawe. 200600007

Rahman, M.M., Younes, H., Subramanian, N., Ghaferi, A.A. Optimizing the dispersion conditions of SWCNTs in aqueous solution of surfactants and organic solvents, Journal of Nanomaterials, 2014, 1-11 (2014). https://doi.org/10.1155/2014/102621

Safi, S., Karimzadeh, F., Labbaf, S. Mesoporous and hollow hydroxyapatite nanostructured particles as a drug delivery vehicle for the local release of ibuprofen, Materials Science and Engineering: C, 92, 712-719 (2018). https://doi.org/10.1016/j. msec.2018.07.004
Sellaoui, L., Guedidi, H., Knani, S., Reinert, L., Duclaux, L., Lamine, A.B. Application of Statistical Physics Formalism to the Modeling of Adsorption Isotherms of Ibuprofen on Activated Carbon, Fluid Phase Equilibria, 387, 103-110 (2015). https://doi. org/10.1016/j.fluid.2014.12.018

Slowing, I.I., Trewyn, B.G., Giri, S., Lin, V.S.Y. Mesoporous silica nanoparticles for drug delivery and biosensing applications, Advanced Functional Materials, 17, 1225-1236 (2007). https://doi. org/10.1002/adfm.200601191

Stuart, B. Infrared Spectroscopy: Fundamentals and Applicationsp203, JohnWiley\&Sons, Ltd, Hoboken (2004). https://doi.org/10.1002/0470011149

Sun, Y., Zhu, L., Wu, T., Cai, T., Gunn, E.M., Yu, L. The nucleation and growth of calcium phosphate by amelogenin, Journal of Crystal Growth, 204, 407-415 (2007). https://doi.org/10.1016/j.jcrysgro.2007.02.035

Szurkowska, K., Kolmas, J. Hydroxyapatites enriched in silicon - Bioceramic materials for biomedical and pharmaceutical applications, Progress in Natural Science: Materials International, 27, 401-409 (2017). https://doi.org/10.1016/j.pnsc.2017.08.009

Tantra, R. Nanomaterials characterization, An Introduction, Chapter 7 Surface Area, John Wiley \& Sons, Inc., New Jersey, Canada (2016).

Tarasevich, B.J., Howard, C.J., Larson, J.L., Snead, M.L., Simmer, J.P., Pain, M., Shaw, W.J. The nucleation and growth of calcium phosphate by amelogenin, Journal of Crystal Growth, 204, 407-415 (2007). https://doi.org/10.1016/j.jcrysgro.2007.02.035

Tomoda, K., Ariizumi, H., Nakaji, T., Makino, K. Hydroxyapatite Particles as Drug Carriers for Proteins, Colloids and Surfaces B: Biointerfaces, 76, 226-235 (2010). https://doi.org/10.1016/j. colsurfb.2009.10.039

Visalli, G., Curro, M., Iannazzo, D., Pistone, A., Pruiti Ciarello, M., Acri, G., Testagrossa, B., Bertuccio, M.P., Squeri, R., Di Pietro, A. In vitro assessment of neurotoxicity and neuroinflammation of homemade MWCNTs, Environmental Toxicology and Pharmacology, 56, 121-128 (2017). https://doi. org/10.1016/j.etap.2017.09.005

Wang, M.C, Shih, W.J., Hung, I.M., Chen, H.T., Hon M.H., Huang, H.H. Characterization of Calcium Phosphate Apatite with Variable $\mathrm{Ca} / \mathrm{P}$ Ratios Sintered at low Temperature, Ceramics International, 41, 1223-1233 (2015). https://doi. org/10.1016/j.ceramint.2014.09.051

Xie, L., Beyer, S., Vogel, V., Wacker, M.G., Mantele, W. Assessing the Drug Release from Nanoparticles: Overcoming the Shortcomings of Dialysis by Using Novel Optical Techniques and a Mathematical Model, International Journal of Pharmaceutics, 488, 108-119 (2015). https://doi.org/10.1016/j. ijpharm.2015.03.080 
Yoon, I.K., Hwang, J.Y., Seo, J.W., Jang, W.C., Kim, X.W., Shin, U.S. Carbon Nanotube-GelatinHydroxyapatite Nanohybrids with Multilayer Core-Shell Structure for Mimicking Natural Bone, Carbon, 77, 379-389 (2014). https://doi. org/10.1016/j.carbon.2014.05.041
Zhao, H.Y., Xu, X.X., Zhang, J.X., Zheng, W., Zheng, Y.F. Carbon nanotube-hydroxyapatitehemoglobin nanocomposites with high bioelectrocatalytic activity, Bioelectrochemistry, 78, 124-129 (2010). https://doi.org/10.1016/j. bioelechem.2009.08.009 